\title{
COMPOSIÇÃO FLORÍSTICA DA ARBORIZAÇÃO DA CIDADE DE ALTAMIRA, PARÁ.
}

\author{
Maurício Möller Parry ${ }^{1}$; Maristela Marques da Silva²; Iracirema Silva Sena ${ }^{3}$; Francisco \\ Plácido Magalhães Oliveira ${ }^{4}$
}

(recebido em 27.06.2011 e aceito para publicação em 15.03.2012)

\section{RESUMO}

Foi realizado em 2010, um inventário da arborização da cidade de Altamira, Pará. Foram amostradas todas as vias públicas dos 25 bairros, com a utilização de uma planilha estruturada para a realização do censo total das espécies presentes nas vias. Foram identificadas 4.294 plantas de 120 espécies, de 105 gêneros e distribuídas em 40 famílias botânicas. A espécie mais frequente foi o ficus (Ficus benjamina), com um total de 1.062 espécimes que representaram $24,7 \%$ das árvores da cidade. A segunda espécie mais frequente foi o macharimbé (Cenostigma macrophyllum) com 793 espécimes (18,5\% do total). Também aparecem a mangueira (Mangifera indica) com 376 espécimes (8,8\%), 0 jambeiro (Syzygium malaccense) com 345 espécimes $(8,0 \%)$, a palmeira imperial (Roystonea oleracea (Jacq.) O. F. Cook) com 135 espécimes (3,1\%), a murta (Murraya paniculata), com 103 espécimes (2,4\%) e o cajueiro (Anacardium occidentale) com 85 espécimes $(2,0 \%)$. As famílias que apresentaram maior número de espécies foram a Fabaceae com 27 espécies, a Arecaceae com 10, Anacardiaceae com 6 e as famílias Malvaceae, Myrtaceae, Moraceae e Bignoniaceae apresentaram cada uma delas, 5 espécies. A grande diversidade de espécies encontradas resulta da participação efetiva da população na arborização da cidade de Altamira.

Palavras-chave: inventário quantitativo, avaliação, arborização urbana.

\footnotetext{
${ }^{1}$ Engenheiro Agrônomo, Professor Doutor da Faculdade de Ciências Biológicas - FCB/UFPA. Altamira, PA. E-mail: mauricioparry@yahoo.com.br

${ }^{2}$ Engenheira Agrônoma, Professora Mestre da Faculdade de Engenharia Agronômica - FEA/UFPA, Altamira, Pará. E-mail: stela@ufpa.com

${ }^{3}$ Engenheira Agrônoma, Pesquisadora Mestre em Fitossanidade da Faculdade de Engenharia Agronômica - FEA/UFPA. Altamira, PA. E-mail: mr.maravilha1@gmail.com ${ }^{4}$ Engenheiro Agrônomo, Professor Doutor da Faculdade de Ciências Biológicas - FCB/UFPA. Altamira, PA. E-mail: placidomagalhaes@yahoo.com.br
} 


\section{FLORISTIC COMPOSITION OF THE URBAN TREES OF ALTAMIRA, PARA.}

\section{ABSTRACT}

An inventory of the urban forest of the city of Altamira, Pará was accomplished. The whole public ways of 25 neighborhoods were sampled with the use of questionnaires. Thus producing a total census of the present species in the streets. There were 4,294 plants sampled of 120 species, 105 genus distributed in 40 botanical families. The most frequent species was the ficus (Ficus benjamina L.), with a total of 1,062 specimens. They represented $24.7 \%$ of the city's trees. The second more frequent species was the macharimbé (Cenostigma macrophyllum) with 793 specimens (18.5\% of the total). Mangueira (Mangifera indica) was also found with 376 specimens $(8.8 \%)$, followed by the jambeiro (Syzygium malaccense) with 345 specimens (8.0\%), imperial palm tree (Roystonea oleracea (Jacq.) O. F. Cook) with 135 specimens (3.1\%), murta (Myrtus communis), with 103 specimens $(2.4 \%)$ and cashew tree (Anacardium occidentale) with 85 specimens $(2.0 \%)$. The families that presented larger number of species were Fabaceae with 27 species, Arecaceae with 10 species, Anacardiaceae with 6 species and the families Malvaceae, Myrtaceae, Moraceae and Bignoniaceae introduced each one of them with 5 species. The major diversity of species found results mainly of the effective participation of the population in tree planting in Altamira city.

Keywords: quantitative inventory, Amazonia, diversity, urban plants

\section{INTRODUÇÃO}

Entende-se por arborização urbana, a vegetação predominantemente arbórea presente em uma cidade, cultivada em áreas particulares (jardins e quintais), praças, parques e vias públicas (SANCHOTENE, 1994).

As árvores encontradas nas áreas livres públicas ou as que acompanham o sistema viário exercem função de corredor ecológico, interligando áreas vegetadas dentro e fora do perímetro urbano, no sentido de melhoria do ambiente, como purificação do ar, melhoria do microclima da cidade, redução da velocidade do vento, abrigo a fauna e amortecimento do 
ruído (RODRIGUES et al., 2002). Em menores proporções, as plantas de menor porte também contribuem com estas funções, na falta da vegetação natural, mantém a cobertura vegetal que protege o solo contra a erosão, mantendo também sua fertilidade e umidade (DENICH, 1989; KÜRTEN, 1998).

A arborização de Altamira começou a ser realizada de forma espontânea por seus moradores antes da década de 70, quando eram plantadas principalmente árvores frutíferas como as Mangueiras (Mangifera indica L.) dentre outras espécies, na frente das casas. No final da década de 1970 e início da década de 1980 foram plantadas muitas espécies exóticas, principalmente as Acácias (Cassia sp.) e as Castanholas (Terminalia catappa L.) nas ruas centrais da cidade (UMBUZEIRO, 1999).

A partir do ano 2000, o processo de expansão da arborização se intensificou, sendo implantadas novas espécies, nativas e exóticas, como os Ipês amarelo e rosa (Handroanthus sp.), o Nim (Azadirachta indica A. Juss), a Acácia-mangio (Acacia mangium Willd.) e o Cajueiro (Anacardium occidentale L.), entretanto, muitas delas ainda inadequadas para este fim e plantadas em locais impróprios, pois as normas técnicas não são de domínio público. Nesta fase, ocorreu um crescimento urbano acelerado e desordenado da cidade, com a criação de novos bairros sem o mínimo de planejamento, surgindo muitos conflitos com a arborização.

As vésperas da cidade de Altamira completar 100 anos em seis de novembro de 2011, foi realizado pela Secretaria de Meio Ambiente e Turismo (SEMAT), juntamente com as instituições de ensino superior do Estado do Pará como a Universidade Federal do Pará (UFPA), Universidade do Estado do Pará (UEPA) e o Instituto Federal do Pará (IFPA) e o Ministério Público Estadual, um inventário da arborização, com a justificativa de se avaliar o estado em que se encontra a arborização e utilizar seus resultados como base para o planejamento urbano do município. O objetivo do presente estudo foi analisar a composição florística da arborização de Altamira, aproveitando a realização do inventário concluído em 2010.

\section{MATERIAIS E MÉTODOS}

A cidade de Altamira está localizada em uma região de ocupação antiga (século XVIII) e que teve um grande impulso recente, incentivada e iniciada pelo governo federal no início da década de 70 (século XX), através do Projeto de Integração Nacional - PIN e de 
um processo de colonização dirigida. Assim, de uma cidade com menos de 15 mil habitantes em 1970, a cidade passa por um crescimento desordenado, sem planejamento de médio e longo prazo, chegando aos atuais 105 mil habitantes (IBGE, 2010).

A cidade de Altamira está localizada nas seguintes coordenadas geográficas: 0312'00" S e 52ำ13'45" W. Fica na margem esquerda do rio Xingu, a 74 metros de altitude, distante da capital do Estado (Belém), $512 \mathrm{~km}$ via aérea, $920 \mathrm{~km}$ via rodovia e $1.857 \mathrm{~km}$ via fluvial (MOURA; RIBEIRO, 2009).

Os solos predominantes na cidade de Altamira são o Neossolos flúvicos localizados nos bairros que margeiam o rio e sofrem a influência das cheias até as avenidas centrais da cidade (Independente II, São Sebastião, Centro e Uirapuru), uma associação dos solos Latossolo Amarelo/Argissolo Vermelho amarelo nos bairros que dominam as encostas da paisagem em forma de manchas nos bairros Independente I, Ibiza, Sudam I e Brasília, e o Nitossolo Vermelho (Terra Roxa) nos bairros que se encontram no topo da paisagem, como os bairros Jardim Ipê, Jardim Altamira, Jardim Oriente e o Bela Vista, classificados de acordo com o Sistema Brasileiro de Classificação dos Solos (EMBRAPA, 2006).

Segundo a classificação Köppen, o clima do município de Altamira é do tipo equatorial Am e Aw. O primeiro, predominante na parte norte do município, onde se localiza a sede municipal, apresentando temperaturas médias de $27,3^{\circ} \mathrm{C}$. A média anual das temperaturas máximas chega a $32,4^{\circ} \mathrm{C}$ e a média das mínimas a $22,1^{\circ} \mathrm{C}$. A precipitação pluviométrica anual gira em torno de $2.123 \mathrm{~mm}$ (SILVA et al., 2009). Devido às elevadas precipitações pluviométricas mensais, a umidade relativa média do ar também é elevada, ficando próximo a 84\% o ano inteiro. O período de menor umidade relativa, com significativa diminuição das chuvas, vai de julho a novembro, já o excedente de água ocorre entre fevereiro e abril.

O inventário foi realizado pela Secretaria Municipal de Meio Ambiente e Turismo (SEMAT) e financiado pelo Ministério Público Estadual, através do censo total das espécies vegetais presentes em todas as vias públicas dos 25 bairros que compõem a sede municipal. Os censos, que incluem a enumeração de todos os indivíduos na área de estudo, têm custo elevado e demandam tempo maior, só se justificam nas avaliações de pequenas áreas ou comunidades (SILVA et al., 2007). O trabalho foi desenvolvido de setembro de 2009 a novembro de 2010, com a participação de docentes da Universidade Federal do Pará (UFPA) como coordenadores, Universidade Estadual do Pará (UEPA) e do Instituto Federal do Pará (IFPA) e alunos destas instituições como voluntários, com o intuito de 
diagnosticar a arborização da cidade assim como do estado geral em que se encontravam as plantas.

Os voluntários receberam orientações durante um curso de capacitação sobre os seguintes temas: botânica; fitossanidade; normas técnicas de urbanismo e arborização; e legislação.

Foram utilizadas planilhas estruturadas para a realização do censo que continham informações sobre as árvores (nome vulgar), fase de desenvolvimento, fitossanidade (com diferentes graus de severidade do ataque), problemas com a raiz (afloramento ou não), necessidade de poda (caso as copas estejam em conflito com a fiação, iluminação ou sinalização pública e necessidade de poda de manutenção), afastamento predial (muros, casas e meio fio), tamanho das calçadas e áreas livres (canteiros), altura, diâmetro a altura do peito (DAP), situação das copas e fenologia. As informações foram coletas in loco.

Os resultados obtidos estão apresentados da seguinte forma para as espécies: nomes populares e científicos, famílias, origem, número de indivíduos por espécie e densidade relativa (Dr). A Dr é a relação percentual entre o número de indivíduos de uma espécie e o número total de indivíduos de todas as espécies. Foi feito o levantamento, sempre que possível, das áreas de distribuição original das espécies, seguindo as nomenclaturas utilizadas por Costa e Lima e Silva Júnior (2010).

Para levantar e identificar os espécimes e chegar as suas respectivas espécies (nome científico e famílias botânicas) foram utilizados os seguintes procedimentos: utilização de fichários de identificação preparado por especialistas, com fotografias, descrição e nomes vulgares e científicos in loco; quando não encontradas no material de apoio, essas espécies tiveram partes vegetativas e reprodutivas coletadas e dissecadas para posterior identificação; por consulta bibliográfica e por meio de chaves de identificação. Os espécimes que não foram identificados por estes métodos, foram herborizados, segundo técnicas detalhadas por Fidalgo e Bononi (1989), e enviados para os seguintes herbários: IAN da Empresa Brasileira de Pesquisa Agropecuária - EMBRAPA Amazônia Oriental, em

Belém; Herbário MG Murça Pires do Museu Paraense Emílio Goeldi e identificados taxonomicamente por especialistas.

Os espécimes incluídos no inventário foram classificados em famílias, de acordo com o sensu Angiosperm Phylogeny Group II (APG II, 2003), para verificação dos binômios, autores corretos e família. Para evitar possíveis confusões botânicas foi utilizado o banco de dados do Missouri Botanical Garden (MOBOT, 2011) e a confirmação das espécies foram 
realizadas através de consulta ao site Lista de Espécies da Flora do Brasil (FORZZA et al., 2010).

\section{RESULTADOS E DISCUSSÃO}

Com o final do inventário da arborização urbana da cidade de Altamira, constatou-se que a composição florística é formada por 4.294 plantas de diferentes hábitos e idades. As plantas são de 120 espécies diferentes, distribuídas em 105 gêneros pertencentes a 40 famílias botânicas. Dentre as famílias botânicas mais numerosas, a família Fabaceae foi a que registrou o maior número de espécies (27) que correspondem a 22,5\% do total das espécies identificadas na cidade e com 1.098 indivíduos, o que representa 25,6\% das plantas utilizadas na arborização. Outras famílias também representadas foram: Arecaceae com 10 espécies; Anacardiaceae (6); Malvaceae, Moraceae, Myrtaceae e Bignoniaceae com 5 espécies cada.

Houve grande diversidade de espécies presentes nas vias públicas, entretanto, apenas 6 espécies contribuíram com 2.814 plantas o que representa $65,5 \%$ do total de indivíduos utilizados na arborização, foram elas: o ficus (Ficus benjamina) com 1.062 indivíduos; o macharimbé (Cenostigma macrophyllum) com 793; a mangueira (Mangifera indica) 376; o jambeiro (Syzygium malaccense) 345; a palmeira imperial (Roystonea oleracea) 135 e a murta (Murraya paniculata) 103 (Tabela 1). Dentre essas, o ficus corresponde com $24,7 \%$ das plantas existentes na arborização da cidade e está presente em todos os bairros e vias da cidade. Os valores agora demonstrados não são os mais apropriados para a arborização urbana. Santamour Júnior (1990) recomenda que não se utilize mais do que $10 \%$ de árvores de uma mesma espécie, $20 \%$ de espécies de um mesmo gênero e 30\% de espécies de uma mesma família, o que não foi observado em Altamira. Para o autor estas regras evitam a propagação de pragas e doenças entre as plantas. 
Tabela 1. Espécies vegetais, famílias botânicas, origem, número de indivíduos e densidade relativa de espécies incluídas no inventário de Altamira, Pará, 2010

Table 1. Vegetation species, botanical families, origin, number of individuals and relative density of species included in the inventory of Altamira, Pará, 2010

\begin{tabular}{|c|c|c|c|c|}
\hline $\begin{array}{l}\text { Espécies } \\
\text { (nome vulgar e científico) }\end{array}$ & Famílias & Origem & $\begin{array}{l}\text { Número } \\
\text { de } \\
\text { Plantas }\end{array}$ & Dr \\
\hline Ficus (Ficus benjamina L.) & Moraceae & $\mathrm{E}$ & 1.062 & 24,73 \\
\hline Macharimbé (Cenostigma macrophyllum & Fabaceae & & & \\
\hline Tul.) & Caesalpinioideae & $\mathrm{Am}$ & 793 & 18,47 \\
\hline $\begin{array}{l}\text { Mangueira (Mangifera indica L.) } \\
\text { Jambeiro (Syzygium malaccense (L.) }\end{array}$ & Anacardiaceae & $E$ & 376 & 8,76 \\
\hline Merr \& L. M. Perry) & Myrtaceae & $\mathrm{E}$ & 345 & 8,03 \\
\hline $\begin{array}{l}\text { Palmeira-imperial (Roystonea oleracea } \\
\text { (Jaca.) O. F. Cook) }\end{array}$ & & $E$ & 135 & 3,14 \\
\hline Murta (Murraya paniculata (L.) Jack) & Myrtaceae & $\mathrm{E}$ & 103 & 2,40 \\
\hline Cajueiro (Anacardium occidentale L.) & Anacardiaceae & $\begin{array}{c}\mathrm{Am} \\
\mathrm{Ma}, \mathrm{Mg}\end{array}$ & 85 & 1,98 \\
\hline Ipê-amarelo (Handroanthus serratifolius & & $\mathrm{Am}, \mathrm{Ce}$ & & \\
\hline $\begin{array}{l}\text { (Vahl.) S. O. Grose) } \\
\text { Nim (Melia indica (A. Juss.) Brandis) }\end{array}$ & $\begin{array}{l}\text { Bignoniaceae } \\
\text { Meliaceae }\end{array}$ & $\underset{\mathrm{E}}{\mathrm{Mg}}$ & $\begin{array}{l}83 \\
80\end{array}$ & $\begin{array}{l}1,93 \\
1,86\end{array}$ \\
\hline Duranta (Duranta repens L. 'aurea') & Verbenaceae & $\mathrm{E}$ & 80 & 1,86 \\
\hline Açaizeiro (Euterpe oleraceae Mart.) & Arecaceae & $\mathrm{Am}$ & 71 & 1,65 \\
\hline Goiabeira (Psidium guayava L.) & Myrtaceae & $\mathrm{Mg}$ & 68 & 1,58 \\
\hline Acacia-mangio (Acacia mangium Willd.) & $\begin{array}{l}\text { Fabaceae } \\
\text { Mimosoideae }\end{array}$ & $\mathrm{E}$ & 66 & 1,54 \\
\hline Oiti (Liconia tomentosa Benth.) & Chrysobalanaceae & $\mathrm{Ca}$ & 50 & 1,16 \\
\hline Pião-roxo (Jatropha gassgpiifolia L.) & Euphorbiaceae & $\mathrm{E}$ & 45 & 1,05 \\
\hline Coqueiro (Cocus nucifera L.) & Arecaceae & $\mathrm{Am}, \mathrm{Ma}$ & 44 & 1,02 \\
\hline Brasileirinha (Erithrina indica Picta) & $\begin{array}{c}\text { Fabaceae } \\
\text { Papilionoideae }\end{array}$ & $E$ & 39 & 0,91 \\
\hline $\begin{array}{l}\text { Castanhola (Terminalia catappa L.) } \\
\text { Areca-bambu (Dypsis lutescens } \mathrm{H} \text {. }\end{array}$ & Combretaceae & $E$ & 37 & 0,86 \\
\hline Wendl.) Beentje \& J. Dransf.) & Arecaceae & $E$ & 33 & 0,77 \\
\hline $\begin{array}{l}\text { Mamoeiro (Carica papaya L.) } \\
\text { Chapéu-de-napoleão }\end{array}$ & Caricaceae & $E$ & 28 & 0,65 \\
\hline peruviana (Pers.) Schum.) & Apocynaceae & $E$ & 24 & 0,56 \\
\hline Tento (Ormosia paraensis Ducke) & $\begin{array}{c}\text { Fabaceae } \\
\text { Papilionoideae }\end{array}$ & $\mathrm{Am}$ & 24 & 0,56 \\
\hline Párkia (Parkia platycephala Benth.) & $\begin{array}{l}\text { Fabaceae } \\
\text { Mimosoideae }\end{array}$ & Am & 24 & 0,56 \\
\hline $\begin{array}{l}\text { Flamboyant-de-jardim } \\
\text { pulcherrima (L.) Sw.) }\end{array}$ & $\begin{array}{c}\text { Fabaceae } \\
\text { Caesalpinioideae }\end{array}$ & $E$ & 23 & 0,54 \\
\hline Bouganville (Bougainvillea spectabillis & Nicte & $E$ & 23 & 0.54 \\
\hline Laranjeira (Citrus sinensis (L.) Osbeck) & Rutaceae & $\mathrm{E}$ & 22 & 0,51 \\
\hline
\end{tabular}


Ameixeira (Syzygium jambolanum (Lam.) DC.)

Cacaueiro (Theobroma cacao L.)

Papoula (Hibiscus rosa-sinensis L.)

Ingá-cipó (Inga edulis (Mart.) Kunth)

Limoeiro (Citrus lemon (L.) Burm. f.)

Sibipiruna (Caesalpinia pluviosa DC.)

Ipê-rosa (Handroanthu ipe (Mart ex K.

Schum.) Standl.

Jasmim-bogari (Jasminum sambac (L.)

Aiton.)

Carambola (Averrhoa carambola L.)

Flamboyant (Delonix regia (Bojer ex

Hook) Raf.

Resedá (Lagerstroemia indica Lam.)

Jasmim-da-India (Quisqualis indica L.)

Algodão (Gossypium hirsutum L.)

Palmeira-rabo-de-peixe (Caryota urens

L.)

Cica (Cycas circinalis L.)

Leucena (Leucena leucocephala (Lam.)

R. de Wit)

Esponjinha (Calliandra brevipes Benth.)

Jasmim-manga (Plumeria rubra L.)

Palheteira (Clitoria racemosa Benth.)

Viuvinha (Petrea subserrata Cham.)

Amoreira (Morus nigra L.)

Ipezinho-de-jardim (Tecoma stans (L.)

Juss. ex Kunth)

Palmeira-leque (Livistona chinensis ( $\mathrm{N}$.

J. Jacquin) R. Brown ex. Mart.)

Cuieira (Crescentia cujete L.)

Tamarindo (Tamarindus indica L.)

Ata (Annona squamosa L.)

Biribá (Rollinia mucosa (Jacq.) Baill)

Munguba (Pachira aquatica Aubl.)

Urucum (Bixa orellana L.)

Cassia-pingo-de-ouro (Cassia fistula L.)

Abacateiro (Persea americana Mill.)

Ixora (Ixora coccinea L.)

Jaca (Artocarpus integrifolia L. f.)

Pata-de-vaca (Bauhinia blakeana Dunn)

\begin{tabular}{|c|c|c|c|}
\hline Myrtaceae & $E$ & 22 & 0,51 \\
\hline Malvaceae & Am & 20 & 0,47 \\
\hline Malvaceae & $E$ & 20 & 0,47 \\
\hline Fabaceae & $\mathrm{Am}$ & 19 & 0,44 \\
\hline \multicolumn{4}{|l|}{ Mimosoideae } \\
\hline Rutaceae & $\mathrm{E}$ & 19 & 0,44 \\
\hline Fabaceae & Ma & 19 & 0,44 \\
\hline \multicolumn{4}{|l|}{ Caesalpinioideae } \\
\hline Bignoniaceae & $E$ & 17 & 0,40 \\
\hline Oleaceae & $E$ & 17 & 0,40 \\
\hline $\begin{array}{c}\text { Oxalidaceae } \\
\text { Fabaceae }\end{array}$ & E & 16 & 0,37 \\
\hline Caesalpinioideae & $E$ & 16 & 0,37 \\
\hline Lythraceae & E & 16 & 0,37 \\
\hline Combretaceae & E & 15 & 0,35 \\
\hline Malvaceae & $E$ & 15 & 0,35 \\
\hline Arecaceae & E & 13 & 0,30 \\
\hline $\begin{array}{c}\text { Cycadaceae } \\
\text { Fabaceae }\end{array}$ & $E$ & 13 & 0,30 \\
\hline Caesalpinioideae & $E$ & 12 & 0,28 \\
\hline $\begin{array}{c}\text { Fabaceae } \\
\text { Mimosoideae }\end{array}$ & $\mathrm{Ma}, \mathrm{Mg}$ & 11 & 0,26 \\
\hline Apocynaceae & $E$ & 11 & 0,26 \\
\hline $\begin{array}{c}\text { Fabaceae } \\
\text { Papilionoideae }\end{array}$ & $\begin{array}{c}\mathrm{Am}, \mathrm{Ce} \\
\mathrm{Mg}\end{array}$ & 10 & 0,23 \\
\hline Verbenaceae & $E$ & 10 & 0,23 \\
\hline Moraceae & $E$ & 9 & 0,21 \\
\hline Bignoniaceae & $E$ & 9 & 0,21 \\
\hline Arecaceae & $E$ & 9 & 0,21 \\
\hline Bignoniaceae & Am & 8 & 0,19 \\
\hline Fabaceae & E & 8 & 0,19 \\
\hline \multicolumn{4}{|l|}{ Caesalpinioideae } \\
\hline Annonaceae & $E$ & 7 & 0,16 \\
\hline Annonaceae & $\begin{array}{c}\mathrm{Am}, \\
\mathrm{Ma}, \mathrm{Ca}\end{array}$ & 7 & 0,16 \\
\hline Malvaceae & Am & 7 & 0,16 \\
\hline Bixaceae & $\mathrm{Am}$ & 7 & 0,16 \\
\hline Fabaceae & $E$ & 6 & 0,14 \\
\hline \multicolumn{4}{|l|}{ Caesalpinioideae } \\
\hline Lauraceae & $E$ & 6 & 0,14 \\
\hline Rubiaceae & $E$ & 6 & 0,14 \\
\hline Moraceae & E & 6 & 0,14 \\
\hline Fabaceae & $E$ & 6 & 0,14 \\
\hline
\end{tabular}


Graviola (Annona muricata L.)

Cacto-mandacaru (Cereus jamacaru P. DC.)

Feijão-guandu (Cajanus cajan (L.) Mill.)

Teca (Tectona grandis L. f.)

Taperebá (Spondias mombin L.)

Pupunheira (Bactris gasipaes Kunth.)

Abiu (Pouteria caimito Ruiz et Pav.)

Ficus-variegata (Ficus benjamina L. var. variegata)

Ipê-verde (Cybistax antisyphilitica (Mart.)

Mart.)

Jucá (Caesalpinia ferrea Mart. ex Tul.

var. ferrea)

Acerola (Malpighia glabra L.)

Mamona (Ricinus communis L.)

Cassia-rosa (Cassia grandis L. f.)

Sena-verrugosa (Senna multijuga (Rich.) Irwin et Barn)

Falso-chorão (Schinus molle L.)

Gmelina (Gmelina arborea Roxb.)

Jarana (Holopydium jarana (Huber)

Ducke)

Mata-pasto (Senna alata (L.) Roxb.)

Mogno (Swietenia macrophylla King.)

Murici (Byrsonima crassifolia (L.) Rich.)

Mussendra (Mussaendra alicia Hort.)

Pião-branco (Jatropha curcas L.)

Pinheiro (Pinus caribaea Morelet)

Tangerina (Citrus sinensis (L.) Osbeck)

Cássia-javanesa (Cassia javanica L.)

Algaroba (Prosopis juliflora (Sw.) DC.)

Ajurú (Chrysobalanus icaco L.)

Alfavaca (Ocimum basilicum L.)

Bambu (Bambusa vulgaris Schrad. ex J.

C. Wendl.)

Bananeira (Musa paradisiaca L.)

Buriti (Mauritia flexuosa L.)

Cajarana (Spondias dulcis Forst.)

Canela (Cinnamomum zeylanicum J.Presl)

Carnaúba (Copernicia prunifera (Miller)
Annonaceae

Cactaceae

Fabaceae

Papilionoideae

Verbenaceae

Anacardiaceae

Arecaceae

Sapotaceae

Moraceae

Bignoniaceae

Fabaceae

Caesalpinioideae

Malpighiaceae

Euphorbiaceae

Fabaceae

Caesalpinioideae

Fabaceae

Caesalpinioideae

Anacardiaceae

Verbenaceae

Lecythidaceae

Fabaceae

Caesalpinioideae

Meliaceae

Malpighiaceae

Rubiaceae

Euphorbiaceae

Pinaceae

Rutaceae

Fabaceae

Caesalpinioideae

Fabaceae

Mimosoideae

Chrysobalanaceae

Lamiaceae

Poaceae

Musaceae

Arecaceae

Anacardiaceae

Lauraceae
Am

$5 \quad 0,12$

$5 \quad 0,12$

$\mathrm{E}$

5

0,12

E

$\mathrm{Am}, \mathrm{Ce}$,

$\mathrm{Ma}$

Am

Am

E

$\mathrm{Ce}$

0,12

$5 \quad 0,12$

$4-0,09$

$4 \quad 0,09$

$4 \quad 0,09$

(1)

$4 \quad 0,09$

Ma

$\begin{array}{lll}\text { E } & 3 & 0,07\end{array}$

$\begin{array}{lll}E & 3 & 0,07\end{array}$

E

0,05

$\begin{array}{lll}E & 2 & 0,05\end{array}$

$\begin{array}{lll}M g & 2 & 0,05\end{array}$

E

0,05

Am

$\mathrm{Am}, \mathrm{Ce}$,

$\mathrm{Ma}, \mathrm{Mg}$

Am

$\mathrm{Am}, \mathrm{Ca}$,

$\mathrm{Ce}$

$\mathrm{E}$
$\mathrm{E}$
$\mathrm{E}$
$\mathrm{E}$
$\mathrm{E}$

$\mathrm{Ca}$

$\mathrm{Am}, \mathrm{Ma}$

Am

0,05

20,05

20,05

20,05

$2 \quad 20,05$

E 20,05

20,05

$2 \quad 0,05$

10,02

$\begin{array}{lll} & 1 & 0,02\end{array}$

10,02

$-1,0,02$

$\begin{array}{lll}\text { E } & 1 & 0,02\end{array}$

10,02

Ce, Am, $\quad 1 \quad 0,02$

$\mathrm{Mg}$

E

0,02

E

$1 \quad 0,02$ 
H. E. Moore

Castanheira (Bertholletia excelsa Kunth)

Cipreste-comum

sempervirens L.)

Dama-da-noite (Epiphyllum oxipetalum

(DC.) Haworth)

Dracena (Dracaena marginata Lam.)

Embaúba (Cecropia adenopus Mart. ex

Miq.)

Espirradeira (Nerium oleander L.)

Eucalipto (Eucalyptus globulus Labil)

Faveiro (Balizia pedicellaris (DC.)

Barneby \& J. W. Grimes)

Fruta-de-macaco (Andira sp.)

Fruta-pão (Artocarpus incisa L.)

Girassol (Helianthus annuus L.)

Guariroba (Syagrus oleracea (Mart.)

Becc.)

Jatobá (Hymenaea courbaril L.)

Ingá-do-mato (Inga vera Willd.) subsp.

affinis (DC.) T.D. Penn

Jurubeba (Solanum paniculatum L.)

Limão (Citrus limon (L.) Burm. f.)

Pombinho (Discophora guianensis

Miers.)

Pau-ferro (Caesalpinia leiostachya

(Benth.) Ducke

Pitomba (Talisia esculenta (St. Hil)

Radlk)

Rosa-madeira (Pereskia grandifolia

Haw.)

Sabugueiro (Sambucus nigra L.)

Sapucaia (Lecythis pisonis Camb.)

Siriguela (Spondias purpurea L.)

Samaumeira (Ceiba pentandra

Gaertn

Trombeta (Datura candida Pasq.)

Espécie desconhecida

(L.)

$\begin{array}{cccc}\text { Arecaceae } & \mathrm{Ca} & 1 & 0,02 \\ \text { Lecythidaceae } & \mathrm{Am} & 1 & 0,02 \\ & & 1 & 0,02 \\ \text { Cupressaceae } & \mathrm{E} & & \\ & \mathrm{Am}, & 1 & 0,02 \\ \text { Cactaceae } & \mathrm{Ma}, \mathrm{Ce} & 1 & 0,02 \\ \text { Ruscaceae } & \mathrm{E} & \end{array}$

$\begin{array}{cccc}\text { Urticaceae } & \text { Am, Mg } & 1 & 0,02 \\ \text { Apocynaceae } & \text { E } & 1 & 0,02 \\ \text { Myrtaceae } & \text { E } & 1 & 0,02\end{array}$

Fabaceae

Mimosoideae

Fabaceae

Caesalpinioideae

Moraceae

Asteraceae

Arecaceae

Fabaceae

Caesalpinioideae

Fabaceae

Mimosoideae

Solanaceae

Rutaceae

Icacinaceae

Fabaceae

Caesalpinioideae

$\mathrm{Am}, \mathrm{Ma}$

0,02

$\mathrm{Am}, \mathrm{Ce}$

$\begin{array}{lll}\text { E } & 1 & 0,02\end{array}$

E

$\mathrm{Ca}, \mathrm{Ce}$,

$\mathrm{Ma}$

Am, Ce

0,02

Am, Ce,

$\begin{array}{lll}\mathrm{Ma} & 1 & 0,02\end{array}$

Am, $\mathrm{Ca} \quad 1 \quad 0,02$

$\mathrm{E}$

0,02

Am, $\mathrm{Ce} \quad 1 \quad 0,02$

Sapindaceae

Cactaceae

Adoxaceae

Lecythidaceae

Anacardiaceae

Malvaceae

Solanaceae
$\mathrm{Ma}$

$\mathrm{Am}, \mathrm{Ma}$

Am, Ma

$\mathrm{Ma}$

$\mathrm{Am}, \mathrm{Mg}$

$\mathrm{Ca}$

Am

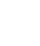

\section{TOTAL GERAL 120 ESPÉCIES}

40 FAMÍLIAS

$-$

$4.294 \quad 100,0$

$\mathrm{Dr}=$ Diversidade relativa; Origem: $\mathrm{Am}=$ Amazônia; $\mathrm{Ca}=$ Caatinga; $\mathrm{Ce}=$ Cerrado; $\mathrm{E}=$ Exótica; $\mathrm{Ma}=\mathrm{Mata}$ Atlântica; $\mathrm{Mg}=$ Mata de galeria.

Durante o processo de coleta e estudo dos dados sobre as espécies plantadas, ficou evidente a participação direta dos moradores na introdução das árvores na cidade. $\mathrm{A}$ quantidade de espécies frutíferas, geralmente, árvores de grande porte como: a mangueira (Mangifera indica) com 376 árvores; o jambeiro (Syzygium malaccense) com 345; o cajueiro 
(Anacardium occidentale) com 85; o açaizeiro (Euterpe oleraceae) com 71; a goiabeira (Psidium guayava) com 68 e o coqueiro (Cocus nucifera) com 44 árvores, que possuem características morfológicas (formato de copa, tamanho e troca das folhas, frutos grandes e sistema radicular superficial) inadequadas para o plantio em vias públicas, principalmente as que apresentam posteamento das redes de fiação elétrica e telefônica, demonstrando as preferências da população por essas árvores. Tais espécies frutíferas representam $23 \%$ do total das plantas encontradas na cidade (Tabela 1).

Poucas foram as espécies nativas somente da Amazônia (13,3\%) utilizadas na arborização urbana de Altamira, muito provavelmente, devido grande parte de a população ser oriunda de outras regiões do Brasil que nela chegaram com o evento da abertura da Rodovia Transamazônica (BR 230), na década de 70 (século XX). Se considerar as espécies nativas encontradas tanto na Amazônia como em outras regiões do Brasil, este número chega a 30,8\%, o que ainda é mínimo em se tratando de biodiversidade amazônica.

Foram observados muitos conflitos (60\%) entre as espécies utilizadas na arborização com as construções (muros, casas e calçadas) assim como, as fiações públicas, iluminação e sinalização das vias (17,3\%). A falta de conhecimento das recomendações técnicas e da legislação urbana por grande parte da população levou ao plantio indiscriminado de espécies impróprias pelas características fisiológicas (plantas tóxicas) e inadequadas pelas suas características morfológicas. Outros fatores que contribuem para estes conflitos são os espaços livres, em forma de canteiros, pequenos demais e principalmente as calçadas que apresentaram em sua maioria (39\%), largura insuficiente para o plantio de árvores de grande porte.

Dentre as dez espécies que ocasionaram o maior número de conflitos nas vias públicas de Altamira, destaca-se o ficus (Ficus benjamina), que também é a mais frequente nas ruas, com total de 1.062 espécimes, o que representa $28,8 \%$ das plantas inventariadas na cidade (Tabela 2). A mangueira com 376 árvores e o jambeiro com 345 também aparecem como as responsáveis por grande parte (78\%) dos serviços de manutenção como as podas e os reparos técnicos. 
Tabela 2. Espécies que apresentaram maior freqüência de conflitos nas vias públicas da cidade de Altamira, Pará, 2010

Table 2. Species that presented larger frequency of conflicts in the public ways of the city of Altamira, Pará, 2010

\begin{tabular}{|c|c|c|c|}
\hline $\begin{array}{l}\text { Espécies } \\
\text { (nome vulgar e científico) }\end{array}$ & Famílias & $\begin{array}{l}\text { Número de } \\
\text { Plantas }\end{array}$ & $\begin{array}{l}\text { Tipos de } \\
\text { Conflitos* }\end{array}$ \\
\hline Ficus (Ficus benjamina L.) & Moraceae & 1.062 & $1,2,3,4,5$ \\
\hline $\begin{array}{l}\text { Mangueira (Mangifera indica L.) } \\
\text { Jambeiro (Svzyaium malaccense (L.) }\end{array}$ & Anacardiaceae & 376 & $1,3,4$ \\
\hline Merr \& L. M. Perry) & Myrtaceae & 345 & $1,2,3,4$ \\
\hline Nim (Melia indica (A. Juss.) Brandis) & Meliaceae & 80 & 1,3 \\
\hline Açaizeiro (Euterpe oleraceae Mart.) & Arecaceae & 71 & 1,3 \\
\hline Acacia mangio (Acacia mangium Willd.) & $\begin{array}{c}\text { Fabaceae } \\
\text { Mimosoideae }\end{array}$ & 66 & 1,3 \\
\hline Coqueiro (Cocus nucifera L.) & Arecaceae & 44 & $1,2,3$ \\
\hline Castanhola (Terminalia catappa L.) & Combretaceae & 37 & $1,3,4$ \\
\hline Tento (Ormosia paraensis Ducke) & $\begin{array}{c}\text { Fabaceae } \\
\text { Papilionoideae }\end{array}$ & 24 & $1,4,5$ \\
\hline Párkia (Parkia platycephala Benth.) & $\begin{array}{c}\text { Fabaceae } \\
\text { Mimosoideae }\end{array}$ & 24 & $1,4,5$ \\
\hline
\end{tabular}

Duas mil quinhentas e uma plantas $(58,2 \%$ do total) se encontram próximas às casas ou aos muros e aos meio fios. A maioria das árvores das espécies contidas na Tabela 2 (1.800 árvores ou 41,9\%) apresentou afloramento de raízes, danificando as calçadas onde se encontram. A poda é o manejo necessário em 574 árvores (13,4\%) devido seus conflitos com a fiação, iluminação e sinalização pública. Para Pivetta e Silva Filho (2002) as características da fiação são importantes fatores a serem observados no planejamento da arborização urbana e deve ser instalada somente de um lado da via, o que normalmente não foi observado em Altamira.

As injúrias ocasionadas por pragas e doenças foram freqüentes e observadas em 2.319 plantas (54\%), sendo que no fícus as infestações foram as mais severas. Além das espécies citadas na Tabela 2, por ocasionarem algum tipo de prejuízo às instituições (públicas ou privadas), deve-se chamar a atenção especial também para o chapéu-denapoleão (Thevetia peruviana), por apresentar látex que em contato com a pele ou ser ingerido principalmente por crianças, pode ser tóxico. Estas espécies devem ser substituídas ou suprimidas gradativamente por espécies mais eficientes nos seus serviços ambientais e urbanísticos. 
A espécie que mais obteve aceitação, tanto por parte das Secretarias de Meio Ambiente como de Agricultura, assim como por grande parte da população, foi o macharimbé (Cenostigma macrophyllum), que tem seu desenvolvimento inicial bastante acelerado e lento quando adulto possui copa densa com floração no ápice dos ramos. Dentre as espécies mais numerosas, ela é a única nativa e já conta com 793 árvores plantadas (18,5\% do total), na sua maioria, plantas jovens. Outra bem sucedida experiência foi a introdução do ipê amarelo (Handroanthus serratifolius), que é uma espécie nativa, entretanto, com número mínimo de árvores (83), o que representa somente 1,9\% do total inventariado na cidade. Outras espécies nativas da região que podem ser mais difundidas são o oiti, a andira-uchi (Andira parviflora) e a palheteira (Clitoria racemosa).

A arborização da cidade não é uniforme, nos bairros centrais foram localizadas e identificadas o maior número de plantas em relação aos periféricos. Este fato não significa que houve prioridade para aqueles em detrimento aos periféricos e sim, está relacionado com o tempo de ocupação das áreas. A arborização dos bairros centrais se deu na década de 70 onde as árvores apresentam em média $50 \mathrm{~cm}$ de circunferência a altura do peito (CAP) e já os periféricos são mais recentes, do final da década de 80 e 90 do século XX, com árvores apresentando CAP de menos de $30 \mathrm{~cm}$ de CAP. Na Tabela 3 estão identificados os bairros com a maior quantidade de indivíduos plantados em suas vias.

Tabela 3. Número de plantas encontradas por bairro, nas vias públicas da cidade de Altamira, Pará, 2010

Table 3. Number of plants found by neighborhood, in the public roads of the city of Altamira, Pará, 2010

\begin{tabular}{lclc}
\hline BAIRROS & $\begin{array}{c}\text { Número de } \\
\text { Plantas }\end{array}$ & BAIRROS & $\begin{array}{c}\text { Número de } \\
\text { Plantas }\end{array}$ \\
\hline Jardim Independente I & 623 & lbiza & 71 \\
Uirapuru & 521 & Liberdade & 55 \\
Centro & 450 & Colina & 51 \\
Brasília & 447 & Jardim Altamira & 44 \\
Sudam I & 396 & Santana & 43 \\
Jardim Independente II & 256 & Sudam II & 43 \\
Mutirão & 253 & Jardim do Sol & 15 \\
Premem & 228 & Jardim Primavera & 15 \\
Aparecida & 206 & Jardim Oriente & 13 \\
São Sebastião & 180 & Bela Vista & 8 \\
Alberto Soares & 167 & Campos Cordeiro & 2 \\
Esplanada do Xingu & 126 & Parque Ipê & 1 \\
Boa Esperança & 80 & & $\mathbf{4 . 2 9 4}$ \\
\hline Subtotal & $\mathbf{3 . 9 3 3}$ & Total Geral & \\
\hline
\end{tabular}


Estes números demonstram que não houve planejamento para a arborização dos bairros novos da cidade, os quais devem ser priorizados em campanhas de plantio futuros, como Rocha et al. (2004) destacaram em seus estudos, já que o conhecimento das suas funções social e ambiental são pré-requisitos básicos para o planejamento e administração das áreas urbanas o que permite, consequentemente, maior eficiência na tomada de decisões (SENNA et al., 2001) na busca de melhores condições de vida para os seus habitantes.

\section{CONCLUSÕES}

A grande diversidade de espécies encontradas resulta da participação efetiva da população na arborização da cidade de Altamira.

Algumas espécies têm que ser substituídas gradativamente das vias públicas por ocasionarem algum tipo de conflito com sinalização, iluminação ou calçadas ou por serem susceptíveis a pragas e doenças como o ficus ou tóxica como o chapéu-de-napoleão.

O macharimbé é a espécie que pode ser melhor difundida na cidade, por ser nativa da região, possuir porte médio e não proporcionar manutenção intensiva nem ocasionar conflitos.

Há a necessidade de ações conjuntas entre as diferentes Secretarias Municipais priorizam a arborização urbana dos bairros da periferia da cidade.

\section{AGRADECIMENTOS}

Agradecemos à Secretaria de Meio Ambiente e Turismo de Altamira e ao Ministério Público Estadual pela oportunidade de trabalharmos em conjunto e aos estudantes do ensino superior (universitários e técnicos) que atuaram ativamente nas ruas da cidade durante as coletas de campo e na tabulação dos dados. Todos foram muito importantes para a realização deste trabalho. 


\section{REFERÊNCIAS}

ANGIOSPERM PHYLOGENY GROUP (APG). An update of the angiosperm phylogeny group classification for the orders and families of flowering plants: APG II. Botanical Journal of the Linnean Society, v. 141, n. 4, p. 399-436, 2003.

COSTA E LIMA, R. M.; SILVA JÚNIOR, M.C. Inventário da arborização urbana implantada na década de 60 no Plano Piloto, Brasília, DF. Revista Brasileira de Arborização Urbana, Piracicaba, SP: v.5, n.4, p.110-127, 2010.

$\mathrm{DENICH}, \mathrm{M}$. Estudo da importância de uma vegetação secundária nova para o incremento da produtividade do sistema de produção na Amazônia Oriental Brasileira. Universidade Georg-August de Göttingen, Alemanha. 1989. 284 p. Tese (Doutorado)

EMPRESA BRASILEIRA DE PESQUISA AGROPECUÁRIA - EMBRAPA. Sistema Brasileiro de Classificação dos Solos. Editores Técnicos, Humberto Gonçalves dos Santos et al. 2 ed. Rio de Janeiro: EMBRAPA Solos, 2006. 306p.

FIDALGO, O.; BONONI, V. L. Técnicas de coleta, preservação e herborização de material botânico. Governo do Estado de São Paulo. Secretaria do Meio Ambiente. Instituto de Botânica. São Paulo. p. 22-24. 1989.

FORZZA, R. C.; LEITMAN, P. M.; COSTA, A. F.; et al. 2010. Introdução. in Lista de Espécies da Flora do Brasil. Jardim Botânico do Rio de Janeiro. Disponível em: <http://floradobrasil.jbrj.gov.br/2010. Acesso em 20 de abril de 2011.

INSTITUTO BRASILEIRO DE GEOGRAFIA E ESTATÍSTICA - IBGE. Cidades@2010. Disponível em: <http://www.ibge.gov.br/home/estatistica/populacao/contagem2010.pdf> Acesso em: 10 de fevereiro de 2011.

KÜRTEN, L. Research for the tropical forest. Ufkes Druck GmbH, Köln: Federal Ministry of Education, Science, Research and Technology, 1998. 88 p. 
MISSOURI BOTANICAL GARDEN (MOBOT). 2011. Disponível em: <http://www.mobot.org/W3T/Search/vast.html.>. Acesso em: 14 de março de 2011.

MOURA, A. R.; RIBEIRO, J. C. Altamira no contexto geográfico. Belém, PA: Ed. Do Autor, 2009. 119p.

PIVETTA, K. F. L.; SILVA FILHO, D. F. Arborização Urbana. Boletim Acadêmico. Jaboticabal: UNESP. FCAV/FUNEP. 2002. 74p.

ROCHA, R. T., LELES, P. S. S., OLIVEIRA NETO, S. N. Arborização de vias púbicas em Nova Iguaçu, RJ: o caso dos bairros Rancho Novo e Centro. Revista Árvore, Viçosa, v. 28, n.4, p. 599-607, julho-agosto. 2004.

RODRIGUES, C. A. G.; BEZERRA, B. C.; ISHII, I. H.; CARDOSO, E. L.; SORIANO, B. M. A.; OLIVEIRA, H. Arborização urbana e produção de mudas de essências florestais nativas em Corumbá, MS. Corumbá: Embrapa Pantanal, 2002. 26p. (Documentos, 42)

SANCHOTENE, M. C. C. Desenvolvimento e perspectivas da arborização urbana no Brasil. In: Congresso Brasileiro de Arborização Urbana, 2, 1994. São Luís, MA. Anais. São Luís, MA. 1994.

SANTAMOUR JÚNIOR, F.S. Trees for urban planting: diversity unifomity, and common sense. In: METRIA Conference, 7., 1990, Lisle. Proceedings... Lisle: p.57-66. 1990.

SENNA, D.; DEUSDARÁ FILHO, R.; PEREIRA, H. S. Arboles fuera del bosque - país: Brasil. FAO/MMA, Brasília, 2001. 13p.

SILVA, A. G.; GONÇALVES, W.; PAIVA, H. N. Avaliando a arborização urbana. Viçosa: Aprenda Fácil, 2007. 346 p.

SILVA, C. S.; AUGUSTO, S. G.; ANDRADE, A. U. Caracterização agrometeorológica de Altamira, PA. SEMANA DE INTEGRAÇÃO DAS CIÊNCIAS AGRÁRIAS, UFPA, IX Altamira, PA: Anais... p. 148-154. 2009.

UMBUZEIRO, Ubirajara Marques. Altamira e sua história. $3^{\mathrm{a}}$. ed. Altamira, 1999. 210p. 